\title{
Pratiques de danse et discours de genre, une histoire connectée
}

\section{Elizabeth Claire}

\section{OpenEdition}

1 Journals

\section{Édition électronique}

URL : https://journals.openedition.org/clio/13682

DOI : 10.4000/clio.13682

ISSN : 1777-5299

Éditeur

Belin

Édition imprimée

Date de publication : 1 décembre 2017

Pagination : 7-18

ISBN : 978-2-410-00859-3

ISSN : 1252-7017

\section{Référence électronique}

Elizabeth Claire, "Pratiques de danse et discours de genre, une histoire connectée », Clio. Femmes, Genre, Histoire [En ligne], 46 | 2017, mis en ligne le 09 avril 2018, consulté le 30 avril 2022. URL : http:// journals.openedition.org/clio/13682; DOI : https://doi.org/10.4000/clio.13682 


\section{Pratiques de danse et discours de genre, une histoire connectée}

Elizabeth CLAIRE

En 1797, un article du Journal des Luxus und der Moden fustige une nouvelle pratique de danse, «bacchanale» prisée par les habitantes de Breslau qui pivotent «comme une figure androgyne déformée » où les pieds «suppriment toute beauté » avec leur «enthousiasme ivre » ${ }^{1}$. Quelques années plus tard, dans ses «Lettres d'un médecin», le rédacteur en chef de la Gazette de santé déplore une forme de lutte entre les sexes qui touche à l'«envie des femmes» (leurs désirs et leurs passions) et nuit à l'État républicain français. Il s'agit du «luxe privé » de la valse, une «contagion générale» de dansomanie venue d'Allemagne qui saisit la capitale parisienne où le couple dansant s'enlace en pivotant « regards confondus, absorbés l'un dans l'autre $»^{2}$. Des médecins ralliés à la cause de l'hygiéniste observent également dans cette danse «moderne » un ébranlement des passions responsable des « renversement[s] de l'ordre général et des lois de la nature », et la fabrique de «femmes hommasses $»^{3}$ attirées par la gloire et l'ambition. Tout le contraire du jugement des écrivaines de l'époque romantique, telle la salonnière berlinoise Rahel Levin Varnhagen qui voit dans la valse «l'art idéal» capable de promouvoir dans le monde entier une sociabilité égalitaire et révolutionnaire ${ }^{4}$. Un siècle plus tard, Nijinski signe la première de L'Après-midi d'un faune au Théâtre du Châtelet, chorégraphie "moderne » des Ballets russes dans laquelle il interprète le personnage du faune, assumant une masculinité androgyne, hétérodoxe

\footnotetext{
Anon. 1797 [traduction de l'auteure].

2 Saint-Ursin 1804 ; cf. Claire 2013a.

3 Dance $1811: 15$.

4 Claire 2013b : 228 ; Tewarson 1998 : 135-136.
} 
et exotique ${ }^{5}$. La représentation fait scandale, le "pantomime trop expressif» de Nijinksi est dénoncé comme «inconvenant avec de vils mouvements de bestialité érotique » suscitant de « justes sifflets » d'un public parisien enflammé ${ }^{6}$. Quelques mois après, les Français sont à nouveau bousculés par une danse «moderne » excessivement érotique et «sauvage », une forme « exotique » de danse de couple importée de l'Amérique du Sud. L'archevêque de Lyon constate le "progrès croissant des mœurs païennes et le fléchissement des mœurs chrétiennes» dans cette danse «lascive» qui conduit la société parisienne jusqu'à l'« état de manie »7

\section{Une pratique de corps excessifs?}

Depuis le Moyen Âge, des légendes comme celle des danseurs maudits de Kölbigk (en Saxe), forcés à «danser en chantant sans repos toute une année » jusqu'à leur libération par l'archevêque de Cologne ${ }^{8}$, participent à l'élaboration en Europe d'un imaginaire liant danses incontrôlables et « accès de folie ». Les médecins naturalisent comme un effet de «l'imagination blessée ${ }^{9}$ des femmes, le phénomène de possession dont les symptômes s'expriment par des mouvements corporels excessifs et incontrôlables. S'ajoutent à ces lieux communs, des stéréotypes construits sur l'image des «pieds ailés » incessants de «l'Africain sauvage» qui «n'a d'autres maîtres que sa passion $»^{10}$. Depuis les premiers contacts des missionnaires européens avec les peuples africains, tout un imaginaire anthropologique racialisant transforme les discours moralisants sur les femmes européennes. Une science des tempéraments, qui pense détecter une "mobilité innée » des femmes, des enfants et des " peuples du sud » ${ }^{11}$, émerge dès la fin

\footnotetext{
5 Garafola 1999 : 246.

6 «Un faux pas », Le Figaro, 30 mai 1912 : 3 ; Garafola 1999.

7 Jacotot 2013 : 355 ; Décoret-Ahiha 2004 : 63-107 ; Semaine Religieuse du Diocèse de Lyon, 1913 : 115-116, cité dans Buch $2017: 217$.

8 Belgrano $2017: 25-27$.

9 Mandressi $2006: 35-70$.

10 Francesco da Roma 1648 et Gioia 1669 : 401-402, cité dans Arcangeli 2017 : 75 76, cf. aussi Arcangeli 2011 ; Voïart 1823 : 214-215.

11 Claire 2013a.
} 
du XVII siècle pour expliquer ce qui amène "les personnes du sexe » involontairement à "l'excès de la danse $»^{12}$. Voilà une hiérarchie de genre renforcée par l'inscription d'une alliance «naturelle» avec des peuples indigènes colonisés.

\section{Comment écrire sur la danse?}

Les exemples de danses passionnées et de leur réception en tant que «manies » montrent que les acteurs historiques ne pouvaient parler de cet art sans évoquer le corps de l'individu - son désir, ses vulnérabilités, sa sensualité, son érotisme, son intimité, sa santé, son androgynie, sa sexualité, son identité, son exotisme, son dérèglement - et ses relations à autrui. Si l'expérience corporelle et émotionnelle de la danse au théâtre semble passer par le regard de l'observateur, elle se saisit également lorsqu'on se livre à la pratique, se formant par la mimèsis et par la répétition des mouvements transmis d'un corps à l'autre, l'expérience du toucher, ou encore par l'effet de la transpiration, de la respiration, des « regards confondus », des rythmes et des gestes partagés. L'expérience de la danse prend sens aussi lorsqu'on tente par l'écriture de saisir ce que dégagent les corps en mouvement, souvent en développant un discours de genre pour faire sens de l'expression corporelle.

Danser ne signifie pas la même chose selon les époques, les aires culturelles ou les groupes sociaux. En fonction de la personne qui énonce une définition de la danse - artiste amateur ou professionnel, écrivain, religieux, médecin, philosophe, politique, parent, chercheur - et du cadre dans lequel il s'exprime, on se trouve face à des expériences de la danse parfois très éloignées. Danser touche aisément aux émotions et aux fondements des liens de sociabilité. Danser veut dire mise en rapport des corps, des passions, des pensées. S'ensuivent, si on se réfère aux sources historiques, des évaluations de la beauté et de la nature des cultures gestuelles, des discours sur les dangers ou la splendeur des chorégraphies, sur la bonté ou les excès d'une forme d'éloquence qui s'exprime sans le recours aux mots. Se pose alors la question épineuse de la lisibilité et

12 Hufeland 1809 : 217-218, 320. 
l'interprétation du corps en mouvement - la danse « comme texte $»^{13}$, la danse «comme performance $»^{14}$, le geste « comme symptôme $»^{15}$, le corps « comme archive $»^{16}$.

\section{De nouvelles approches historiques}

Jusqu'à très récemment, l'histoire de la danse et en particulier «l'historiographie féministe de la danse ${ }^{17}$, s'est concentrée sur les chorégraphies scéniques théâtrales ${ }^{18}$. La contribution importante du numéro thématique de Recherches en danse (2015) consacré à la question du genre et dirigé par Hélène Marquié et Marina Nodera entame une discussion sur la place des femmes dans les danses scéniques des XVIII $^{\mathrm{e}}$ et XIX ${ }^{\mathrm{e}}$ siècles : la collection propose une histoire des danseuses professionnelles issues du contexte théâtral et principalement français. Sans nier l'urgence de ce travail toujours à mener, il parait désormais nécessaire d'élargir la perspective, de s'affranchir des limites de la scène occidentale et de l'analyse des œuvres qui dominent l'historiographie. Ce numéro de Clio. FGH invite précisément à repenser la constitution et l'interrogation de nos sources et à obtenir une vision plus large de ce que représente « la danse ».

La spécificité de l'histoire ici privilégiée s'inscrit dans la continuité d'un travail collectif expérimenté au sein de l'Atelier d'histoire culturelle de la danse ${ }^{19}$ à l'EHESS depuis 2007. Nous souhaitons proposer de nouvelles approches historiques soucieuses de l'interprétation des cultures gestuelles et physiques incorporées par les danses du passé et attachées à l'étude diachronique et connectée de la diversité des périodes, des lieux et des aires culturelles. Une attention particulière est ainsi portée aux pratiques et à la circulation géographique inattendue

\footnotetext{
13 Franko 2015 [1993].

14 Schechner 2012 ; Schechner 2013 : 38-40.

15 McCarren 1998.

16 Cf. Actualités dans ce numéro.

17 Nordera $2007: 172$.

18 Launay et al. 2014.

19 Mes remerciements à Emmanuelle Delattre-Destemberg, Marie Glon, Sophie Jacotot, Vannina Olivesi, Juan Ignacio Vallejos de l'AHCD, et Esteban Buch du CRAL pour leur collaboration [http://ahcdanse.hypotheses.org/].
} 
des discours sur les danses qui informent sur leur réception à l'échelle internationale. Cela permet de repérer des transferts culturels dans l'histoire de cet art, d'ouvrir des passerelles d'analyse, de débusquer des résonances dans les rapports de genre en jeu.

La diversité des danses présentées dans ces textes invite à observer les manifestations de géopolitiques genrées qui s'expriment par l'acte de danser. Il s'agit de dépasser les discours qui nationalisent la culture des danses, tel que celui sur le « rayonnement» mythique de la danse française depuis Louis XIV20. L'idée est d'offrir aux lectrices et lecteurs de Clio. FGH, le plus possible, les résultats d'une recherche collective menée sur cette «embodied social practice ${ }^{21}$. Cette approche permet d'interroger non seulement les œuvres chorégraphiques ou les objets de la culture dite "savante ${ }^{22}$, mais d'explorer aussi tout un ensemble de pratiques, d'objets et de représentations de la danse. Ces recherches conduisent à associer la danse à d'autres pratiques qui la croisent et qui informent sur l'histoire du genre que la danse incorpore. Elles aident à théoriser l'éducation, la religion, la politique, l'esthétique, l'économie, la médecine, la prostitution, la musique, la science, l'art plastique, la religion, le statut d'auteur, les mobilités des praticiens, leurs migrations, exils et diasporas.

Transversale à la plupart des contributions, la question de la moralisation des pratiques de danse permet de présenter les diverses manières par lesquelles les praticien.ne.s ont su se positionner à l'égard de ce que Marie Glon et Juan Ignacio Vallejos (en dialogue avec Foucault) nomment le «problème moral de la danse » qui constitue, au moins dans l'histoire occidentale, " une activité dont la moralité ne va pas de soi - que cette moralité se réfère à des normes religieuses, nationales, civiques, raciales, médicales, à des normes de genre, de classe sociale... $»^{23}$. Comme le montre Rafael Mandressi qui s'intéresse à la première réception parisienne du tango (1907-1914), la question de la morale croise des enjeux politiques. Considéré comme excessivement érotique et sauvage, le tango est condamné par

\footnotetext{
20 Delattre-Destemberg, Glon \& Olivesi 2014.

21 Desmond 1999 : 319-324 ; Desmond 2001 : 3-32.

22 Pouillaude 2009.

23 Glon \& Vallejos 2017 : 9.
} 
l'écrivain Georges Goursat (Sem) qui se lamente sur l'adoption parisienne de cette danse «névrotique» par lequel, «[1]a moitié de Paris frotte l'autre»; d'autres auteurs n'y voient qu'une «lourde pantomime» obscène masculinisant les femmes, féminisant les hommes et reflétant «le tempérament d'un nègre ${ }^{24}$. Or, loin de souligner son érotisme, ses défenseurs proposent une appropriation patriotique de cette danse moderne venue d'ailleurs afin d'assurer que la France garde son rôle de capitale culturelle, et perdure comme « pays où la danse est nécessaire à la vie » ${ }^{25}$.

L'article d'Ida Meftahi offre un exemple inattendu de l'importance de la circulation de cette danse de couple, devenue "parisienne » avant la Grande Guerre et introduite en Iran par le biais de l'occupation militaire des Alliés, événement qui se conjugue avec le Programme Pahlavi pour l'émancipation des femmes en 1936. Une fois adopté à Téhéran, le tango propose à ses citoyens une "méthode parisienne moderne $»^{26}$ de danse qui est dénoncée comme obsédante lorsqu'elle est pratiquée par les femmes de l'élite urbaine. Ce tango suscite en outre des condamnations quant à ses effets sur la masculinité musulmane et l'intégration des femmes iraniennes dans la société moderne.

Cette thématique de la morale est particulièrement pertinente dans les deux études d'iconographie que nous offrent Marina Nordera et Christiane Klapisch-Zuber. Nordera pointe la mobilisation d'images de danseuses dans des estampes représentant l'allégorie biblique des dix vierges (Matthieu $25: 1$-13) produites entre les XVI et XVII siècles aux Pays-Bas. Cette nouveauté dans l'iconographie de la parabole va de pair avec une activité des maitres à danser inédite et une circulation importante en Europe de leurs manuels et traités de danse qui œuvrent à façonner des gestes en proposant des règles pour la retenue, notamment des femmes, correspondant à une intériorisation des normes corporelles de la civilité urbaine et, pour le cas examiné, calviniste. En interrogeant l'opposition symbolique entre «l'image positive des femmes qui travaillent et celle négative des femmes qui

\footnotetext{
24 Sem 1913 et Dubois 1914, cité dans l'article de Rafael Mandressi dans ce numéro.

25 Le Temps, 26 octobre 1913, cité dans Buch $2017: 216$.

26 Baghdasariyan 1937: 7, et Baghdasarian 1940: 13, cité dans l'article de Ida Meftahi dans ce numéro.
} 
dansent » dans les estampes de Breughel, Jean Saenredam et Crispin de Passe, Nordera expose combien le «dérèglement gestuel » des femmes dessiné par ces artistes figure comme une «dissipation collective de forces productives et morales» qui aurait des répercussions sur la société dans son ensemble.

Le jugement moralisant de la figure de Salomé étudié par Christiane Klapisch-Zuber, par contre, est plus ambigu : si aux yeux des religieux et des théologiens, Salomé en train de danser sert le plus souvent d'archétype de la mauvaise femme, l'auteure nous rappelle que la danse à la Renaissance n'est pas systématiquement condamnable. Se demandant si la danse «en solitaire » que la jeune Salomé exécuta devant le roi Hérode Antipas, permet d'exprimer « les pôles genrés de la danse », Klapisch-Zuber propose une lecture des représentations du Banquet d'Hérode produites entre 1200 et 1490 par San Zeno Maggiore, Fra Angelico, Benozzo Gozzoli et Domenico Ghirlandaio. Une lecture subtile de la " gestuelle ralentie et mesurée » ressemblant aux bassa danza de la haute aristocratie au Quattrocento, montre la danse de Salomé qui émerge au début du Cinquecento surtout comme "ambivalente », voire placée au service de l'évocation d'héroïnes bibliques et antiques, si on la considère dans le contexte des réformes religieuses du $\mathrm{XVI}^{\mathrm{e}}$ siècle. Un autre exemple d'une polarité de genre est exposé dans la contribution de Ludmilla Acone qui étudie la danse noble du Quattrocento: sa lecture évoque l'opposition mythique et complémentaire entre les figures de Mars et Vénus théorisée par des maîtres à danser qui font de la danse de cour une pratique corporelle de «vertu guerrière masculine et de beauté et douceur féminine » pour l'instruction des jeunes des familles de l'élite.

Danser est ainsi un dispositif corporel, révélateur des faits ainsi que des troubles sociaux. Elle lie l'action et l'expression du corps aux normes et aux rapports sociaux de genre. Balladine Vialle offre un exemple contemporain avec la danse d'abidous qui sert de rituel d'entrée dans l'âge adulte, pratique qui prend une place privilégiée dans la vie des jeunes générations berbères au Maroc : sous l'effet de la migration en France, des mutations importantes s'opèrent dans la transmission d'expertises musicales et gestuelles liées à cette tradition. Ces changements soulignent toute la complexité de la rencontre d'une masculinité pratiquée et préservée « au pays » par les adolescents qui 
dansent, dès lors qu'ils sont confrontés avec des lieux communs genrés de Français qui considèrent la pratique de la danse comme une affaire « de filles » ${ }^{27}$ ou d'homosexuels.

D'autres exemples des divisions sexuées dans la transmission, la création, et l'interprétation des danses sont explorées dans ce numéro, et permettent de revisiter et de nuancer des oublis historiographiques concernant la contribution féminine à diverses traditions. Vannina Olivesi propose un nouveau point de vue sur la création féminine du ballet romantique à travers une lecture située de la carrière professionnelle de Marie Taglioni. L'auteure restitue les circonstances institutionnelles qui encadrent la production et la «féminisation» de la danse dans les entreprises parisiennes de spectacle au XIX ${ }^{\mathrm{e}}$ siècle, et éclaire les contraintes professionnelles en vigueur à l'Opéra de Paris privilégiant l'autorité et l'auctorialité du maitre de ballet sur la composition chorégraphique des œuvres spectaculaires. On observe l'importance du statut du vedettariat et de la capacité à mobiliser un réseau féminin et mondain à Paris, deux facteurs qui permettent à Taglioni de faire face à son exclusion du statut de «maître », et ainsi vivre de son métier par la création et l'enseignement des danses de société dans des cadres privés et alternatifs.

Interroger, avec un regard de genre, la danse comme un travail ouvre des pistes pour déconstruire la figure mythique de l'auteur singulier qui a largement dominé l'historiographie jusqu'à aujourd'hui. Ce qui permet également de considérer l'importance d'autres pratiques, telles les danses de société qu'enseignait Taglioni, révélatrices des effets du genre mais rarement étudiées. Sarah Olsen présente une lecture de la participation des orchestris dans la culture du symposium en Grèce antique. En interrogeant leurs danses comme du travail professionnel, l'auteure nous permet de sortir des clichés discursifs qui construisent ces figures uniquement comme des objets de l'entreprise prostitutionnelle, où l'analyse de leurs danses n'avait pas d'intérêt particulier. Par l'élucidation des enjeux professionnels qui se manifestent dans le contexte du symposium où le pouvoir de la séduction féminine se confronte à la culture philosophique masculine, l'auteure nous permet également d'évaluer l'importance de la

27 Marquié 2016. 
compétition qui pouvait exister entre ces femmes orchestris et l'« agency» que la danse pouvait leur offrir dans ce contexte.

La thématique du travail en danse est explorée aussi dans l'article de Prarthana Purkayastha qui expose l'apport de Pratima Devi à l'œuvre chorégraphique et au projet pédagogique en danse de son beau-père, le célèbre poète indien Rabindranath Tagore, fondateur d'une université, qui dirige une compagnie de spectacle au Bengale à la fin de la période coloniale. Purkayastha interroge la création chorégraphique collective et féminine qui permet, dans ce contexte, la naissance et la transmission d'une danse moderne, malgré l'influence des luttes féministes et nationalistes en Inde qui ne songeaient pas à une telle place pour les femmes bourgeoises dans la production artistique. L'article souligne en outre l'importance du rôle de la collectivité dans l'acte chorégraphique, valorisant la transmission des savoirs corporels et l'enseignement des techniques et des théories de la danse, activités plus souvent allouées aux femmes, comme éléments essentiels au développement de cet art.

À travers ces divers exemples, nous constatons, avec Mark Franko, Felicia McCarren et bien d'autres, que la danse - l'art du geste - est capable d'interroger, de théoriser, de construire, de déconstruire et de commenter des relations sociales et l'incorporation du genre tout particulièrement ${ }^{28}$. Elle se prête bien à l'engagement du chercheur.e en histoire du genre, et à l'envie de regarder le passé d'un œil critique. Sans pour autant renoncer à la spécificité de la danse en tant qu'expression corporelle, on réclame la possibilité pour cette pratique de prendre place au sein des sciences sociales non seulement comme objet légitime mais aussi comme analyseur de l'histoire. Par l'exploration des diverses " géopoétiques » genrées, le numéro souhaite également inviter à un regard féministe encore désireux d'imaginer autrement notre futur « connecté » ${ }^{29}$.

28 Franko 2015 [1993] ; McCarren 1998 ; cf. Actualité de la recherche dans ce numéro.

29 Je m’inspire des échanges théoriques sur la notion d'histoire connectée, et du projet de critique féministe évoqué par Joan W. Scott lorsqu'elle constate que le désir est un dispositif critique: «Desire moves metonymically; relations among 


\section{Bibliographie}

ANON., 1797 (juin), "Tanzmoden in Breslau, Aus einem Briefe » [Modes de danse à Breslau, d'une Lettre], Journal des Luxus und der Moden, 6, p. 289-292.

ARCANGELI Alessandro, 2011, «Fonti europee e cultura arabo-islamica di fronte ai balli africani: Missionari, viaggiatori, trattatist», in Massimo DonATTINI, Giuseppe Marcocci \& Stefania PASTORE (a cura di), L'Europa divisa e $i$ Nuovi Mondi. Per Adriano Prosperi, vol. II, Pisa, Edizioni della Normale, p. 241-252.

—, 2017, "The Savage, The Peasant and the Witch », in Marie GLON et Juan Ignacio VAllejos (eds), European Drama and Performance Studies. Danse et morale, une approche généalogique, n 8, Paris, Classiques Garnier, p. 71-91.

Belgrano Adrien, 2017, «Danses profanes et lieux sacrés au Moyen Âge Central. Les danses dans les cimetières entre contrôle social et négociations ", European Drama and Performance Studies, $8:$ «Danse et morale, une approche généalogique » (sous la dir. de Marie GLON \& Juan Ignacio VAlLEjos), p. 25-41.

Buch Esteban, 2017, «La Censure du tango par l'église de France à la veille de la Grande Guerre. Avec un post-scriptum d'Erik Satie ", European Drama and Performance Studies, 8 : «Danse et morale, une approche généalogique » (sous la dir. de Marie Glon \& Juan Ignacio VAllejos), p. 211-235.

ClaIRE Elizabeth, 2013a, "Inscrire le corps révolutionnaire dans la pathologie morale : la valse, le vertige, et l'imagination des femmes", Orages. Littérature et culture 1760-1830, 12 : «Sexes en Révolution » (sous la dir. de Florence LOTTERIE, Pierre FranTZ \& Olivier BARA), p. 87-109.

—, 2013b, "Walqliebelust: vertigine e sogno di egualitarismo », in Susanne Franco (dir.), «Immaginari corporei e rappresentazioni di genere tra danza, scrittura e società », Nuove frontiere per la storia di genere, atti del Congresso SIS 2010 (Napoli, 28-30 Genero 2010) Laura Guidi \& Maria Rosaria Pellizzari (a cura di), Padova, Webster Press \& Università di Salerno, p. 223-228.

DANCE Jean Étienne, 1811, De l'Influence des Passions sur la santé des femmes, Paris, Didot Jeune.

DÉCoret-Ahiha Anne, 2004, «La 'dansomanie' exotique », in Anne DéCoreTAhiHA, Les Danses exotiques en France, 1880-1940, Paris, Centre national de la danse, p. 63-107.

its objects are characterized by unexpected contiguities. The movements are lateral, and they don't follow a single direction. We might say here that for feminism, desire is driven by a form of critique - or, better, is itself a critical faculty », cf. Scott $2011: 34$. 
DESMOND Jane, 1999, «Engendering dance: feminist inquiry and dance research », in Sondra Horton Fraleigh \& Penelope Hanstein (eds), Researching Dance: evolving modes of inquiry, Pittsburgh, University of Pittsburgh Press, p. 309-333.

—, 2001, "Making the invisible visible: staging sexualities through dance », in Jane Desmond (ed.), Dancing Desires: choreographing sexualities on and off the stage, Madison, University of Wisconsin Press, p. 3-32.

Delattre-Destemberg Emmanuelle, Glon Marie \& Vannina Olivesi (transl. Elizabeth Claire), 2014, «Review essay: Mathias Auclair et Christophe Ghristi (dir.), Le Ballet de l'Opéra. Trois Siècle de Suprématie Depuis Louis XIV ", Dance Research Journal, 46/1, p. 104-113 [version française : https://ahcdanse.hypotheses.org/38].

Dubois Lucile, 1914, «La France jugée à l'étranger. Le Tango », Mercure de France, $\mathrm{n}^{\circ} 400$, tome CVII, 25e année, 16 février, p. 871-875.

FrANCESCO DA ROMA, Giovanni, 1648, Breve relatione del successo della missione de frati minori Capuccini del serafico P.S. Francesco al regno del Congo e delle qualità, costumi, e maniere di vivere di quel regno, e suoi habitatori, Rome, Sacra Congregatione de Propaganda Fide.

Franko Mark, 2015 [1993] Dance as Text: ideologies of the baroque body, New York, Oxford University Press.

Garafola Lynn, 1999, "Reconfiguring the sexes », in Lynn Garafola \& Nancy Van Norman Baer (eds), The Ballets Russes and its World, New Haven, Yale University Press, p. 245-268.

GiOIA Francesco Maria, 1669, La maravighiosa conversione alla santa fede di Cristo della regina Singa, e del suo regno di Matamba nell'Africa meridionale [...] cavata da una relatione di là mandata dal p.f. Antonio da Gaeta, Napoli, G. Passaro.

Glon Marie \& Juan Ignacio VALlejos, 2017, «La danse est-elle (im)morale? Éléments pour une étude généalogique », European Drama and Performance Studies, $8:$ : Danse et morale, une approche généalogique » (sous la dir. de Marie GLON \& Juan Ignacio VALLEJOS), p. 9-23.

HufELAND Guillaume, 1809 [2nde éd. all., 1798], L'Art de prolonger la vie humaine, traduit [par Auguste Devau], Lausanne, Hignou et Comp.

ЈАсотот Sophie, 2013, Danser à Paris dans l'entre-deux-guerres. Lieux, pratiques et imaginaires des danses de société des Amériques (1919-1939), Paris, Éd. Nouveau Monde.

Launay Isabelle, Clavel Joanne, Jacotot Sophie, Prouteau-Steinhausser Ninon \& Violeta SaLVATIERRA, 2014, «Devenir chercheuses en danse, entretien à plusieurs voix», Recherches en danse, 1, "Être chercheur en danse?» [http://danse.revues.org/516]. 
MANDressi Rafael, 2006, «Les Médecins et le diable. Expertises médicales dans les cas de possession démoniaque au XVII siècle en France ", Chrétiens et sociétés, 13 [http://chretienssocietes.revues.org/2111].

MARQuiÉ Hélène, 2016, Non, la danse n'est pas un truc de filles !, Toulouse, Éd. de l'Attribut.

MARQuiÉ Hélène \& Marina Nordera (dir.), 2015, « Perspectives genrées sur les femmes dans l'histoire de la danse », Recherches en danse, 3 [http://danse.revues.org/837].

MCCARren Felicia, 1998, Dance Pathologies: performance, poetics, medicine, Stanford University Press.

Nordera Marina, 2007, "Gender Underway», in Marina Nordera \& Susanne Franco (eds), Dance Discourses. Keywords in Dance Research, London, Routledge.

Pouillaude Frédéric, 2009, Le Désceuvrement chorégraphique. Étude sur la notion d'cuvre en danse, Paris, Vrin.

SAint-Ursin P.J. Marie, 1804, "Lettre Sixième : Du Luxe privé - de la walse », in L'Ami des Femmes, ou Lettres d'un médecin [...] dédié à Mme. Bonaparte, Paris, Barba, p. 59-68.

ScOTT Joan W., 2011, The Fantasy of Feminist History, Durham, Duke UP.

SCHECHNER Richard, 2013, Performance Studies: An Introduction, New York, Routledge.

—, 2012, "Is/As Performance », in the Routledge Companion Website to Performance Studies - An Introduction [https://youtu.be/SB6zTUfEODc]

Semaine religieuse du Diocèse de Lyon, 26 décembre 1913.

SEM 1913, «Les Possédées », Le Journal, 15 avril, p. 1 ; 28 avril, p. 1 ; 18 mai, p. 1.

Tewarson Heidi Thomann, 1998, Rabel Levin Varnhagen. The Life and Work of a German Jewish Intellectual, Lincoln, Univ. of Nebraska Press.

VOÏART Élise, 1823, Encyclopédie des Dames: Essai sur la danse, Paris, Audot. 